\title{
Achromatic beamline design for a laser-driven proton therapy accelerator
}

\author{
K. D. Wang $\odot$, K. Zhu, ${ }^{*}$ Matthew J. Easton®, Y. J. Li, C. Lin, and X. Q. Yan ${ }^{\dagger}$ \\ State Key Laboratory of Nuclear Physics and Technology, and Key Laboratory of HEDP \\ of the Ministry of Education, CAPT, Peking University, Beijing 100871, China
}

(Received 24 August 2020; accepted 6 November 2020; published 18 November 2020; corrected 2 December 2020)

\begin{abstract}
A new laser-driven proton therapy facility is being designed by Peking University. The protons will be produced by laser-plasma interaction, using a 2-PW laser to reach proton energies up to $100 \mathrm{MeV}$. We hope that the construction of this facility will promote the real-world applications of laser accelerators. Based on the experimental results and design experience of existing devices in Peking University, we propose a beam transmission system which is suitable for the beam produced by laser acceleration, and demonstrate its feasibility through theoretical simulation. It is designed with two transport lines to provide both horizontal and vertical irradiation modes. We have used a locally-achromatic design method with new canted-cosinetheta (CCT) magnets. These two measures allow us to mitigate the negative effects of large energy spread produced by laser-acceleration, and to reduce the overall weight of the vertical beamline. The beamline contains a complete energy selection system, which can reduce the energy spread of the laser-accelerated beam enough to meet the application requirements. The users can select the proton beam energy within the range 40-100 MeV, which is then transmitted through the rest of the beamline. A beam spot with diameter of less than $15 \mathrm{~mm}$ and energy spread of less than $5 \%$ can be provided at the horizontal and vertical irradiation targets.
\end{abstract}

DOI: $10.1103 /$ PhysRevAccelBeams.23.111302

\section{INTRODUCTION}

With the increasing demand for accelerators with higher energies and smaller footprints, the research of novel particle acceleration mechanisms is attracting more attention in the accelerator community. Ultraintense and ultrashort laser-driven accelerators have become one of the most attractive topics in the accelerator community in recent years, due to their high accelerating gradients [1]. Since the first experiments on interactions between high-intensity laser pulses and solid targets, new research into different acceleration regimes has produced some meaningful experimental results [2-7]. Laser-driven accelerators with radiation pressure acceleration (RPA) have generated proton energies up to $93 \mathrm{MeV}$ [8-10]. Such results indicate that laser accelerators could be utilized for various applications. One of the most important applications is radiotherapy. Several facilities for laser-driven proton therapy are being built or in preparation for construction around the world, including ELIMED (the ELI-beamlines medical and multidisciplinary applications) in the EU [11,12], and

\footnotetext{
"zhukun@pku.edu.cn

xueqingyan@pku.edu.cn
}

Published by the American Physical Society under the terms of the Creative Commons Attribution 4.0 International license. Further distribution of this work must maintain attribution to the author(s) and the published article's title, journal citation, and DOI. the Center for Advanced Laser Applications (CALA) in Germany [13].

CLAPA-T is a new proton therapy facility under development by Peking University, based on the research experience of the Compact Laser Plasma Accelerator (CLAPA) [14,15], and supported by China's Ministry of Science and Technology. The layout of the facility and its beam transport system are shown in Fig. 1.

A key technology for CLAPA-T is the near-criticaldensity target [16], which consists of self-sustained foil material and carbon nanotubes. The laser system will be a 2-PW, 1-Hz Ti:sapphire system using chirped pulse amplification (CPA) technology delivering pulses with $60 \mathrm{~J}$ energy, 30 fs duration, and a contrast of $10^{10}$ at 100 ps. Utilizing this petawatt laser system, we will be able to repeatedly generate proton beams with high peak flux and short temporal duration on the energy level of many hundred $\mathrm{MeV}$.

The project will be completed in two phases. The first phase, as described in detail below, is to produce a $100 \mathrm{MeV}$ proton beam with pulses of more than $10^{8}$ particles and continuously adjustable energy with 5\% spread. Such a beam passing through the transmission system can meet many of the therapeutic requirements. In the second phase, the facility will be upgraded to produce $200 \mathrm{MeV}$ proton beams.

Compared with traditional accelerator beams, laserdriven beams have certain distinct characteristics [17-19]: (a) short pulse widths; (b) wide energy spectra; (c) large 


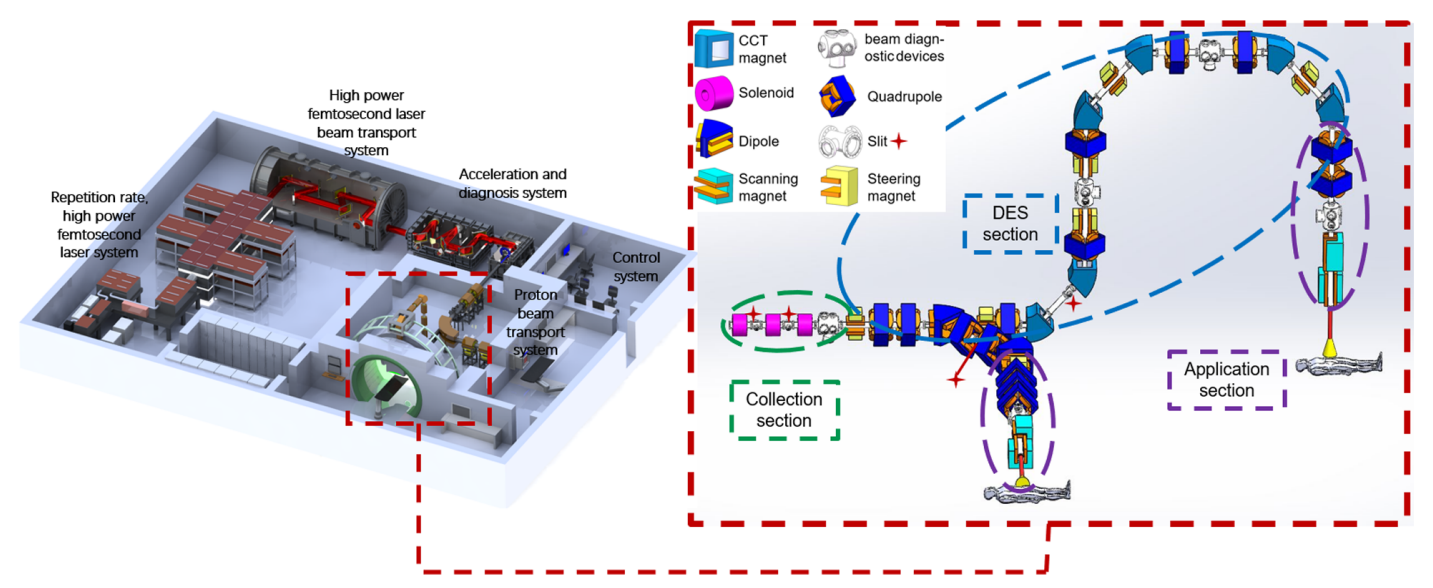

FIG. 1. Layout of CLAPA-T and its transport system. The overall layout of the beam transport system is in the red dotted box. Different sections of the beam transport system are circled by dashed lines of different colors.

angular spread; and (d) a spectrum of secondary particles. Designing a proton therapy system tailored for these characteristics is a challenging task [20].

As experimental data for laser-driven accelerators at the energy range of the CLAPA-T facility are not yet available, we have carried out a careful dynamics design for the beamline, considering operational reliability, flexibility and compactness. We use an achromatic lattice to control the envelope growth caused by the large dispersion and energy spread, and use superconducting solenoids and curved canted-cosine-theta (CCT) magnets to make the vertical beam line lighter and more compact [21], as part of an exploration for the subsequent construction of a superconducting gantry.

\section{MAIN PARAMETERS AND OPTICS DESIGN}

For the dynamics design, we used the TraceWin code [22], which has been used in many laser-driven accelerator transport line designs. This code combines fast beam envelope calculations with full multiparticle simulations to balance efficiency with accuracy.

Considering the above characteristics of laser-driven beams and the requirements for the first phase of the project, we determined the design objectives as follows: (a) proton beams with energy in the range $40-100 \mathrm{MeV}$ can be transmitted, fulfilling the treatment requirements planned for phase 1; (b) beamlines can provide both horizontal and vertical irradiation, to reach tumors in different parts of the body; (c) beam spot with energy spread of less than 5\% and diameter of less than $15 \mathrm{~mm}$ can be formed at the irradiation terminal. These criteria are a compromise between the control of irradiation and characteristics of beams produced by laser acceleration. Although the beam energy generated in this first phase of the project is lower than beam of the traditional accelerator therapy device, it is sufficient to treat some shallow cancers and most childhood cancers [23]. In future, smaller beam spot sizes could also be obtained by using a collimator in front of the patient location.

To meet the different transport requirements, we treat the horizontal and vertical beam transport lines separately. Each entire beamline is required to perform particle collection, transport and delivery. First, the beamline needs to collect the laser-accelerated proton beam with its large angular divergence. Then, the beam transmission process requires energy selection and beam steering. Finally, the beamline in front of the patient location must include beam shaping and matching, as well as beam scanning.

We extrapolate the initial conditions for the beam based on experimental data from laser-generated proton beams at lower energies. Referring to the experimental experience from CLAPA, combined with theoretical simulations, we set the initial transverse radius of the beam spot as $5.0 \mu \mathrm{m}$, which is almost identical to that of the laser spot [24]. We set the initial divergence angle and beam energy spread to $\pm 50 \mathrm{mrad}$ and $\pm 10 \%$ respectively. The distance from the laser target to the first element is $250 \mathrm{~mm}$. For the macroparticle calculations, we model the initial beam with uniform distributions in the 4D transverse space and a Gaussian distribution in energy. Longitudinally, we focus on the selection of energy and control of energy spread rather than on the particular longitudinal distribution. Along the beamline, the longitudinal distribution is almost unchanged, so this can be assumed constant for the rest of the beamline.

\section{A. Beam collection section}

The collection section of the transport system is used to focus the initial large divergence angle beam and make a preliminary energy selection. It consists mainly of three superconducting solenoids [25], which can collect the beam with a wide range of energies and divergence angles. We use the solenoid chromatic effect to control the energy spread [26], by positioning two adjustable apertures between the three solenoids. This arrangement of two 


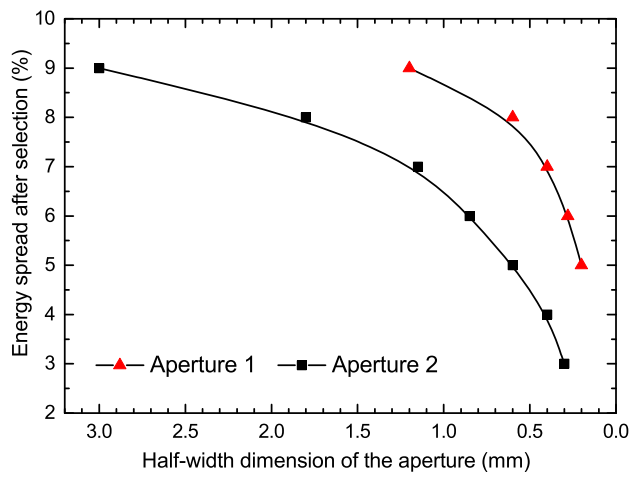

(a)

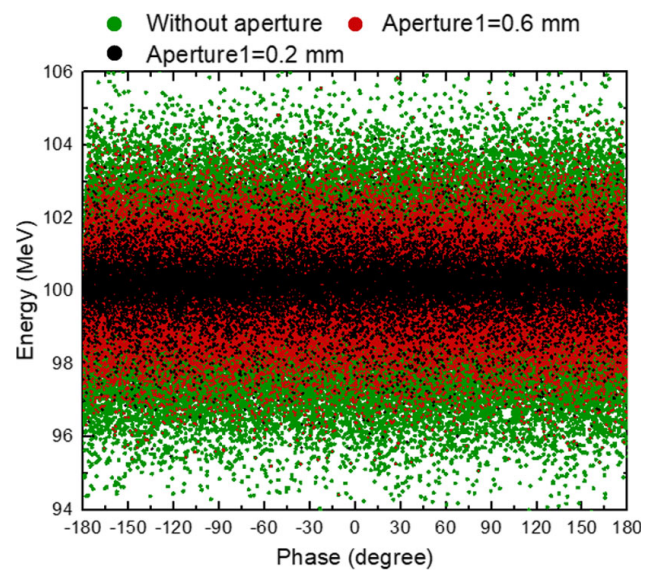

(b)

FIG. 2. Simulation results in the collection section, showing (a) relationship between aperture size and energy selection for the two apertures; and (b) final phase-energy distribution from multiparticle simulation when adjusting the first aperture.

apertures provides a variety of options for controlling situations that may emerge during the operation of the device. Based on our simulations, we expect to mainly make use of the first aperture for control of the energy spread during operation, with the second aperture being held in reserve for extra control if needed. Drawing on the operational experience of CLAPA [24], we simulated the transmission of a beam with 5\% Gaussian energy spread through the aperture to investigate the relationship between aperture size and energy spread selection range.

Figure 2 shows the results of these simulations. Although the distribution of energy of these simulations is not representative of the expected laser-generated spectrum, these simulation results demonstrate the ability to select an energy range and limit the energy spread transmitted to the next section. By adjusting the size of the aperture, particles with incorrect energy can be filtered out. Through this process, we obtain a proton beam with energy spread reduced to approximately $\pm 1 \%$. By the end of the collection section, we can convert the laser-generated beam with a radius of $100 \mu \mathrm{m}$ and divergence angle of $50 \mathrm{mrad}$ to quasiparallel beams with a radius of approximately $5 \mathrm{~mm}$.
During operation, it is the aperture here that will exclude the majority of the unwanted particles.

\section{B. Beam deflection and energy selection (DES) section}

Charged particle therapy requires proton beams with a small, controllable energy spread. This means that the energy selection section is a very important part of a laserdriven accelerator. The deflection and energy selection (DES) section of our transport line accurately selects the treatment energy, and also removes uncharged particles by changing the beam direction. The irradiation depth in the patient tissue can be controlled effectively by selecting the appropriate beam energy. The required energy is selected from a different range of the broad-energy beam produced by the laser acceleration.

The collection section focuses the proton beam to form a beam waist in transverse space at the entrance of the DES section. The beamline splits into separate horizontal and vertical beamlines from the DES section onwards, and the deflection elements in each beamline guide the beam to the desired patient treatment location. The beam conditions after the collection section are listed in Table I. An adjustable slit set between the magnets removes protons with incorrect energies and stray neutral particles produced by laser acceleration.

When particles with an energy deviation pass through the deflecting dipole magnet, their trajectories gain an angular perturbation [27]. Under this influence, the transverse and longitudinal motion of the beam will be coupled together. Although it can be proved that this transverse-longitudinal coupling does not result in the increase of six-dimensional phase space volume, the increase in emittance in the projection space also results in an increase in the beam envelope. This means that the momentum spread of the beam will cause an increase in the transverse beam envelope. This can be expressed as:

$$
a_{x}=\sqrt{a_{x, 0}^{2}+\left(D_{x} \frac{\Delta p}{p}\right)^{2}}
$$

where $a_{x}$ and $a_{x, 0}$ are the beam envelope with and without dispersion respectively, $\Delta p / p$ is the relative momentum deviation, and $D_{x}$ is the dispersion function, which satisfies the equation [27]:

TABLE I. Main beam parameters at the start of the deflection and energy selection (DES) sections.

\begin{tabular}{lrr}
\hline \hline & DES-H & DES-V \\
\hline$\alpha_{x}$ & -0.89 & -0.25 \\
$\beta_{x}(\mathrm{~mm} / \mathrm{mrad})$ & 12.18 & 12.87 \\
$x_{\max }(\mathrm{mm})$ & 7.23 & 7.77 \\
$\alpha_{y}$ & -0.89 & -0.25 \\
$\beta_{y}(\mathrm{~mm} / \mathrm{mrad})$ & 12.18 & 12.87 \\
$y_{\max }(\mathrm{mm})$ & 7.33 & 7.69 \\
\hline \hline
\end{tabular}




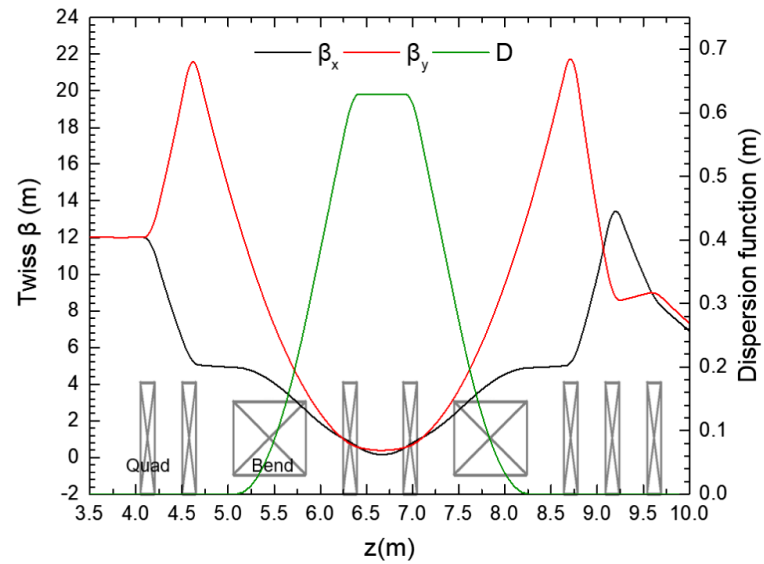

(a)

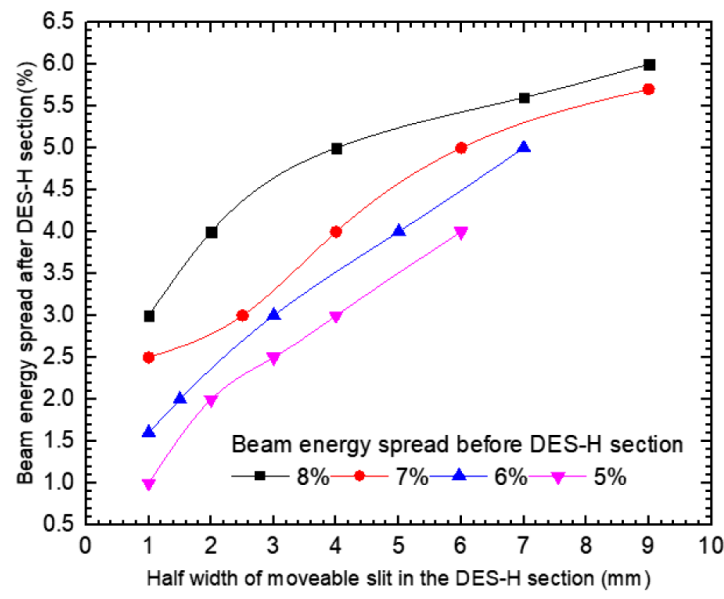

(b)

FIG. 3. Beam parameters in the DES-H section, showing (a) Twiss- $\beta$ and dispersion functions along the $z$ axis; and (b) Final energy spread against slit width for different initial energy spreads.

$$
D^{\prime \prime}+\left[\frac{1}{\rho^{2}} \frac{2 p_{0}-p}{p}+\frac{B^{\prime}}{B \rho} \frac{p_{0}}{p}\right] D=\frac{1}{\rho} .
$$

Here $\rho$ is the bending radius of the dipole magnet and $B$ is its magnetic flux density. As dispersion also makes the envelope of the beam sensitive to its energy spread, we have used an achromatic lattice design. This method can effectively limit the dispersion to local segments to reduce its influence [28-30].

In the horizontal beam line section (DES-H), two $45^{\circ}$ bending magnets and two quadrupole magnets form an overall achromatic beam line. Figure 3(a) shows the change of final Twiss-beta and dispersion functions along the whole horizontal beamline for the final beam envelopes after optimization. The dispersion function emerges at the starting point of the first dipole magnet arc, reaches its maximum value between the two dipole magnets, and drops to zero at the exit of the second dipole. The Twissbeta functions in both the $x$ and $y$ directions decrease with the increase of the dispersion function and reach their minimum values where the dispersion function is maximized. This approach minimizes the effect of dispersion on beam envelope and instability. We have placed a moveable high-precision slit in the middle of DES-H to carry out the fine energy selection and removal of neutral particles. Figure 3(b) shows the simulation results for the relationship between the half-width of the movable slit and the final energy spread. The different colored lines represent simulations with different values for energy spread preselected in the collection section.

In the vertical beam line section (DES-V), we make use of curved canted-cosine-theta (CCT) magnets [21,31,32], which are composed of multiple pairs of alternately tilted solenoid windings. Compared with traditional magnet structures, the inclined solenoid coil structure is lightweight and the magnetic field quality is superior, allowing us to significantly reduce the weight of the vertical beamline. In this project, we will use multiple nested coil pairs in the CCT magnets to produce a hybrid field with both dipole and quadrupole components. The dipole field is distributed throughout the whole length of the CCT magnets, whereas the quadrupole field is applied within a range near the entrance and exit of the CCT magnets, and can be independently adjusted. To simulate this complex field in TraceWin, we use separate 3D field map files for the dipole and quadrupole fields, including fringe fields, and calculate

TABLE II. Main parameters of CCT magnets.

\begin{tabular}{|c|c|c|c|c|c|c|}
\hline & CCT1 & $\mathrm{CCT} 2$ & СCT3 & CCT4 & CCT5 & CCT6 \\
\hline Total length $(\mathrm{mm})$ & 785 & 785 & 785 & 785 & 785 & 785 \\
\hline Bending angle (degree) & 45 & 45 & 45 & 45 & 45 & 45 \\
\hline Bend radius $(\mathrm{mm})$ & 1000 & 1000 & 1000 & 1000 & 1000 & 1000 \\
\hline Aperture $(\mathrm{mm})$ & 36 & 36 & 36 & 36 & 36 & 36 \\
\hline Dipole field strength (T) & -1.48 & -1.48 & 1.48 & 1.48 & 1.48 & 1.48 \\
\hline Dipole length (mm) & 785 & 785 & 785 & 785 & 785 & 785 \\
\hline Inlet quadrupole field gradient $(\mathrm{T} / \mathrm{m})$ & 17.22 & -25.22 & 17.22 & -25.22 & 17.22 & -25.22 \\
\hline Outlet quadrupole field gradient $(\mathrm{T} / \mathrm{m})$ & -25.22 & 17.22 & -25.22 & 17.22 & -25.22 & 18.50 \\
\hline Inlet quadrupole length (mm) & 200 & 200 & 200 & 200 & 200 & 200 \\
\hline Outlet quadrupole length $(\mathrm{mm})$ & 200 & 200 & 200 & 200 & 200 & 200 \\
\hline
\end{tabular}




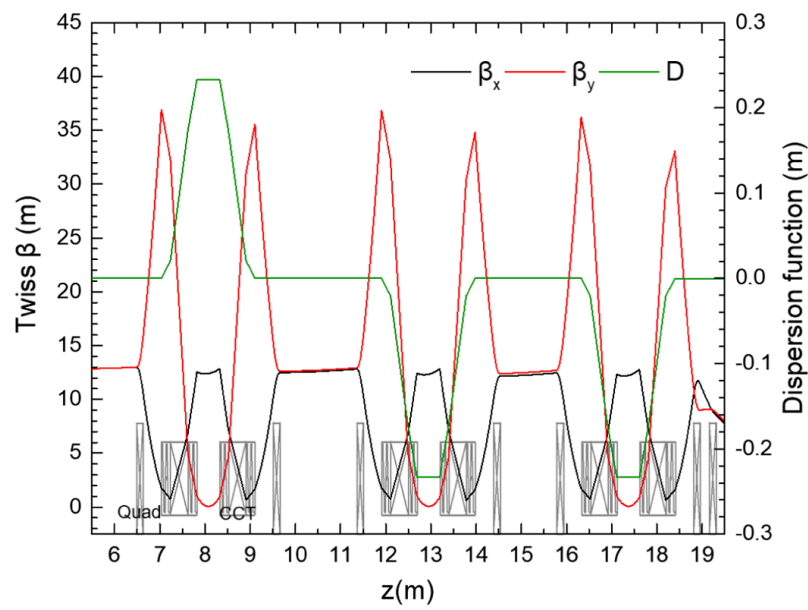

(a)

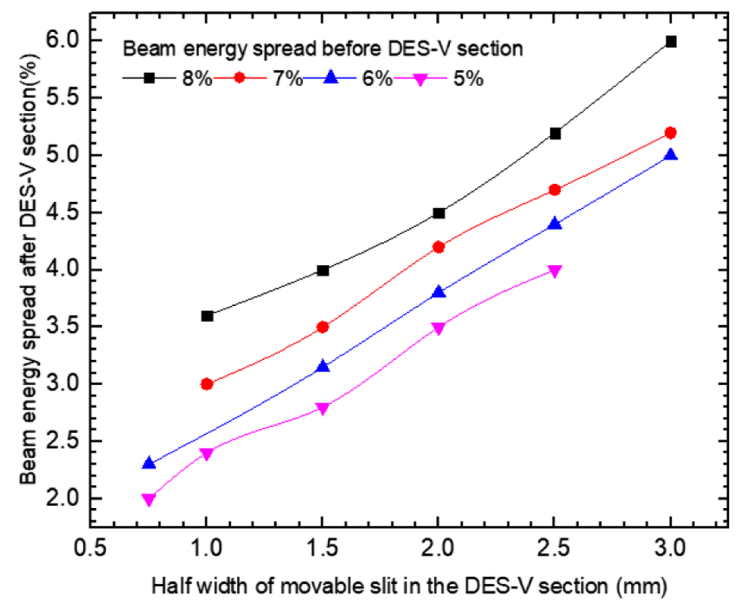

(b)

FIG. 4. Beam parameters in the DES-V section, showing (a) Twiss- $\beta$ and dispersion function along the $z$ axis; and (b) Final energy spread against slit width for different initial energy spreads.

a linear superposition to produce the combined field sections. The dipole-only segments of the CCT magnets use the built-in dipole elements. This is a common method to check the rationality of the magnetic field design in traditional accelerators. The vertical beamline design contains three locally achromatic cells using six of these curved CCT magnets. The main parameters of the CCT magnets are given in Table II.

Figure 4(a) shows the change of the Twiss-beta and dispersion functions through the DES-V section. By adjusting the distribution of the quadrupole field within the CCT, we can constrain the dispersion function to be nonzero only between each pair of neighboring CCT magnets. As in the DES-H section, we use a high-precision slit for further particle selection, located where the beam is first deflected in the DES-V section. Figure 4(b) shows the simulation results for the relationship between and the halfwidth of the DES-V movable slit and the final energy spread.

In summary, the horizontal DES-H section comprises two $45^{\circ}$ bending magnets, four quadrupole magnets and

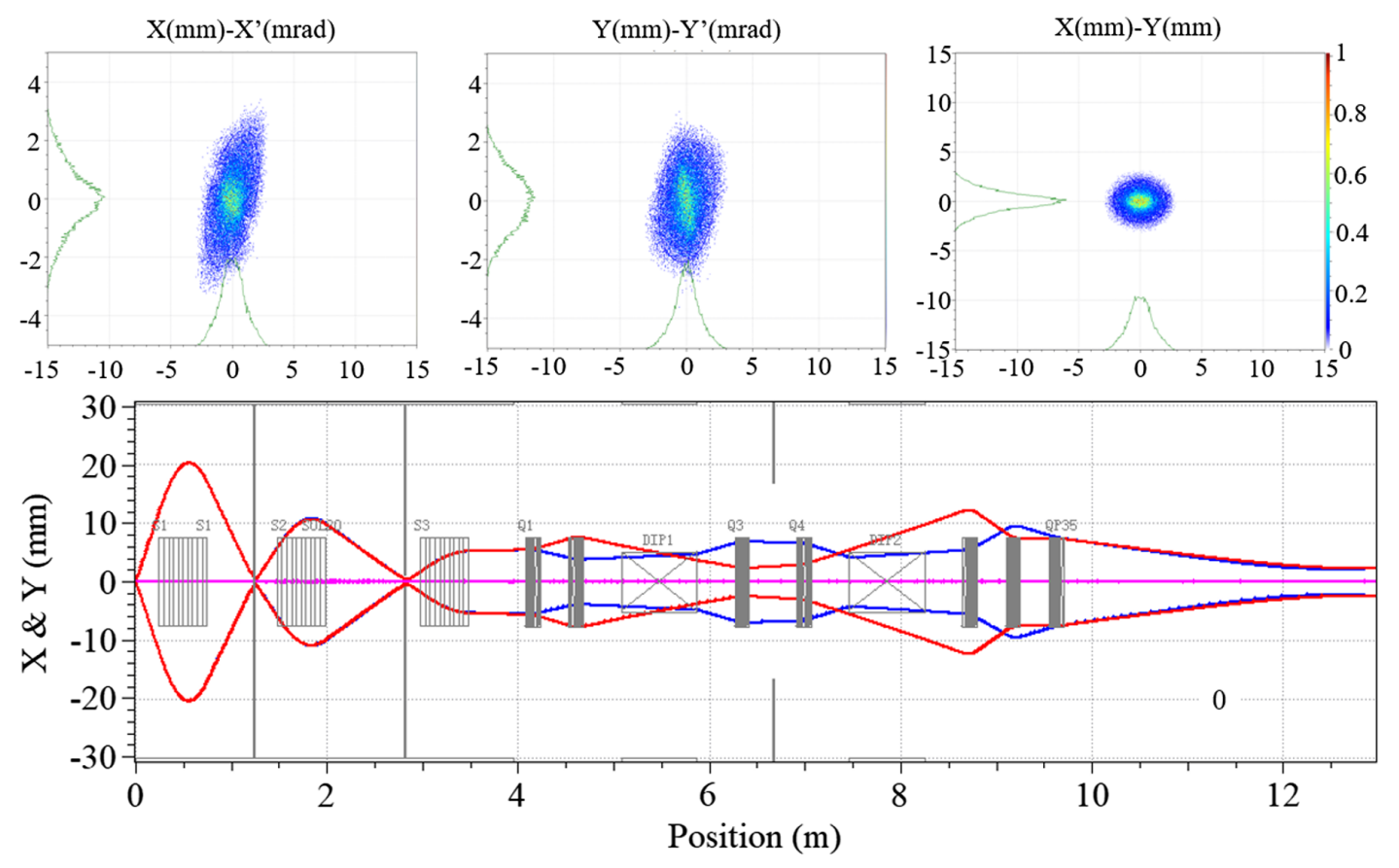

FIG. 5. Upper: simulation results for the transverse phase space at the exit of the horizontal beamline. Plots from left to right are the phase space diagrams in the $x$ and $y$ directions, and a cross-section of the beam at the irradiation point. Lower: beam envelope evolution along the $z$ axis. The blue line represents the $x$-direction, and the red line represents the $y$-direction. 


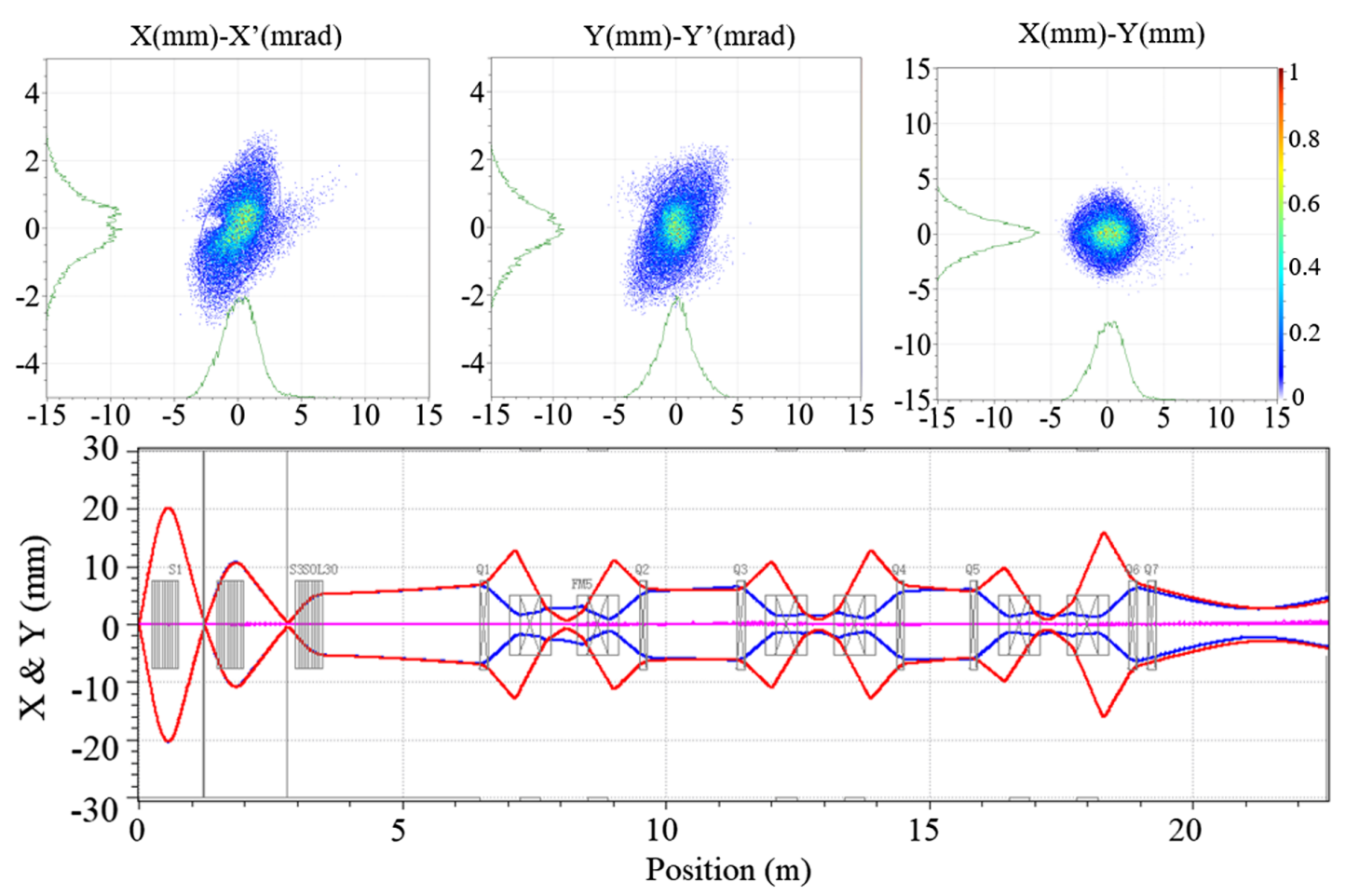

FIG. 6. Upper: simulation results for the transverse phase space at the exit of the vertical beamline. Plots from left to right are the phase space diagrams in the $x$ and $y$ directions, and a cross-section of the beam at the irradiation point. Lower: beam envelope evolution along the $z$ axis. The blue line represents the $x$-direction, and the red line represents the $y$-direction.

one moveable slit. Six $45^{\circ}$ superconducting CCT magnets and one moveable slit constitute the vertical DES-V section. In Fig. 1, the DES section component layout is circled with the blue dotted line.

\section{Application section}

After the DES section, the beam enters the final application section with the correct energy and direction of motion. This final section shapes the beam to be ready for irradiation. We use normal-conducting quadrupole magnets, with two installed in the vertical beamline and three in the horizontal beamline. The application section can tailor the beam to the desired shape when it reaches the irradiation area, and continuously adjusts the beam spot size to keep it within a certain range. Scanning magnets in the $\mathrm{x}$ and $\mathrm{y}$ directions are positioned after the quadrupole to perform spot scanning for radiotherapy. In Fig. 1, the application section component layout is circled with a purple dotted line.

We simulated the beam transmission at full energy using the TraceWin code, with 100000 macroparticles. This simulation allows study of the impact of nonlinear effects. The central energy of the particles is $100 \mathrm{MeV}$, and the energy spread is within 5\%. The expected beam current is $10 \mathrm{nA}$, and for such low currents, the space charge effect can be neglected. We also ran simulations at lower energies in the specified range $40-100 \mathrm{MeV}$, by adjusting the element magnetic field strength according to the ratio of magnetic rigidities. Results were consistent with the full energy simulation results presented here.

Figures 5 and 6 show the simulation results. The beam envelope stays within $20 \mathrm{~mm}$ and varies linearly throughout the entire transmission process. By adjusting the two

TABLE III. Main parameters for the focusing elements of the beam transmission system.

\begin{tabular}{lccccc}
\hline \hline Section & Element & $\begin{array}{c}\text { Length } \\
(\mathrm{mm})\end{array}$ & $\begin{array}{c}\text { Radius } \\
(\mathrm{mm})\end{array}$ & $\begin{array}{c}\text { Max field } \\
\text { or gradient }\end{array}$ \\
\hline Collection & Solenoid 1 & 500 & 30 & 9.05 & $\mathrm{~T}$ \\
& Solenoid 2 & 500 & 30 & 8.23 & $\mathrm{~T}$ \\
& Solenoid 3 & 500 & 30 & 6.51 & $\mathrm{~T}$ \\
DES-H & Quad. 1 & 150 & 36 & 7.90 & $\mathrm{~T} / \mathrm{m}$ \\
& Quad. 2 & 150 & 36 & -11.06 & $\mathrm{~T} / \mathrm{m}$ \\
& Quad. 3 & 150 & 36 & 11.43 & $\mathrm{~T} / \mathrm{m}$ \\
Application-H & Quad. 4 & 150 & 36 & 11.45 & $\mathrm{~T} / \mathrm{m}$ \\
& Quad. 5 & 150 & 36 & -14.11 & $\mathrm{~T} / \mathrm{m}$ \\
DES-V & Quad. 6 & 150 & 36 & 15.07 & $\mathrm{~T} / \mathrm{m}$ \\
& Quad. 1 & 150 & 36 & -3.17 & $\mathrm{~T} / \mathrm{m}$ \\
& Quad. 2 & 150 & 36 & -13.69 & $\mathrm{~T} / \mathrm{m}$ \\
& Quad. 3 & 150 & 36 & -14.41 & $\mathrm{~T} / \mathrm{m}$ \\
& Quad. 4 & 150 & 36 & -13.91 & $\mathrm{~T} / \mathrm{m}$ \\
Application-V & Quad. 5 & 150 & 36 & -14.29 & $\mathrm{~T} / \mathrm{m}$ \\
& Quad. 6 & 150 & 36 & -18.31 & $\mathrm{~T} / \mathrm{m}$ \\
& Quad. 7 & 150 & 36 & 3.07 & $\mathrm{~T} / \mathrm{m}$ \\
\hline \hline
\end{tabular}


quadrupole magnets at the end of the vertical and horizontal beamline correspondingly, we can gather the beam in the transverse space into the required diameters of $5 \mathrm{~mm}$ and $10 \mathrm{~mm}$ respectively. For the longitudinal space, we can keep the energy spread below $5 \%$ by adjusting the first moveable slit in the collection section and the two moveable slits in the DES sections. Table III lists the basic parameters for the focusing elements of the $100 \mathrm{MeV}$ proton beamline: length, aperture and magnetic flux density.

\section{SUMMARY}

In this paper, we have detailed the design of the beamline for CLAPA-T phase 1. This beamline is scheduled to be completed by 2025 . The beamline design includes both horizontal and vertical irradiation beam lines. The length and bending angle of the horizontal beamline are $12.96 \mathrm{~m}$ and $90^{\circ}$, and for the vertical beamline these values are $22.56 \mathrm{~m}$ and $270^{\circ}$ respectively. By adjusting load current of the elements in the beamline, we can transmit proton beams with energies in the range $40-100 \mathrm{MeV}$. We use a variety of energy selection elements to effectively filter both particles with large energy variation and stray neutral particles. We can constrain the final energy spread to stay below 5\% and can continuously adjust the energies within a certain range. Each bending element of the beamline is locally achromatic, which allows us to significantly limit the envelope growth of the beam due to dispersion. The use of CCT magnet technology in the vertical beamline can provide design experience for follow-up work with a superconducting gantry. The next phase of work will focus on the enhancement of the maximum transmission energy and the uniformity of beam profiles. If the whole project is successfully completed, it will play a great role in promoting real applications of laser-driven accelerators.

\section{ACKNOWLEDGMENTS}

This work was jointly supported by the National Key Research and Development Program of China for the development of the proton beam transmission system and proton therapy nozzle (No. 2019YFF01014403), the National Grand Instrument Project (No. SQ2019YFF010006), and the National Natural Science Foundation of China (grants No. 11975037 and No. 11921006).

[1] P. Mulser, D. Bauer, and H. Ruhl, Phys. Rev. Lett. 101, 225002 (2008).

[2] R. H. Hu, B. Liu, H. Y. Lu, M. L. Zhou, C. Lin, Z. M. Sheng, C. E. Chen, X. T. He, and X. Q. Yan, Sci. Rep. 5, 15499 (2015).

[3] S. Zhao, C. Lin, J. E. Chen, W. J. Ma, J. J. Wang, and X. Q. Yan, Chin. Phys. Lett. 33, 035202 (2016).
[4] M. L. Zhou, B. Liu, R. H. Hu, Y. R. Shou, C. Lin, H. Y. Lu, Y. R. Lu, Y. Q. Gu, W. J. Ma, and X. Q. Yan, Phys. Plasmas 23, 083109 (2016).

[5] K. Lan, J. Liu, Z. C. Li, X. F. Xie, W. Y. Huo, Y. H. Chen, G. L. Ren, C. Y. Zheng, D. Yang, S. W. Li et al., Matter Radiat. Extremes 1, 8 (2016).

[6] T. Toncian, C. Wang, E. McCary, A. Meadows, A. Arefiev, J. Blakeney, K. Serratto, D. Kuk, C. Chester et al., Matter Radiat. Extremes 1, 82 (2016).

[7] W. Y. Huo, Z. C. Li, D. Yang, K. Lan, J. Liu, G. L. Ren, S. W. Li, Z. W. Yang, L. Guo, L. F. Hou, X. F. Xie et al., Matter Radiat. Extremes 1, 2 (2016).

[8] Z. C. Yan, W. Nörtershäuser, and G. W. F. Drake, Phys. Rev. Lett. 100, 243002 (2008).

[9] H. Y. Wang, C. Lin, Z. M. Sheng, B. Liu, S. Zhao, Z. Y. Guo, Y. R. Lu, X. T. He, J. E. Chen, and X. Q. Yan, Phys. Rev. Lett. 107, 265002 (2011).

[10] I. J. Kim, K. H. Pae, I. W. Choi, C.-L. Lee, H. T. Kim, H. Singhal, J. H. Sung, S. K. Lee, H. W. Lee, P. V. Nickles, T. M. Jeong, C. M. Kim, and C. H. Nam, Phys. Plasmas 23, 070701 (2016).

[11] G. A. Cirrone, M. Carpinelli, G. Cuttone, S. Gammino, S. B. Jia, G. Korn, M. Maggiore, L. Manti, D. Margarone et al., Nucl. Instrum. Methods Phys. Res., Sect. A 730, 174 (2013).

[12] F. Romano, F. Schillaci, G. Cirrone, G. Cuttone, V. Scuderi, L. Allegra, A. Amato, A. Amico, G. Candiano et al., Nucl. Instrum. Methods Phys. Res., Sect. A 829, 153 (2016).

[13] F. Lindner, D. Haffa, J. Bin, F. Englbrecht, Y. Gao, J. Gebhard, J. Hartmann, P. Hilz, C. Kreuzer, S. Lehrack et al., Nucl. Instrum. Methods Phys. Res., Sect. B 402, 354 (2017).

[14] J. G. Zhu, M. J. Wu, Q. Liao, Y. X. Geng, K. Zhu, C. C. Li, X. H. Xu, D. Y. Li, Y. R. Shou, T. Yang et al., Phys. Rev. Accel. Beams 22, 061302 (2019).

[15] J. G. Zhu, K. Zhu, L. Tao, Y. X. Geng, C. Lin, W. J. Ma, H. Y. Lu, Y. Y. Zhao, Y. R. Lu, J. E. Chen, and X. Q. Yan, Chin. Phys. Lett. 34, 054101 (2017).

[16] A. Yogo, H. Daido, S. V. Bulanov, K. Nemoto, Y. Oishi, T. Nayuki, T. Fujii, K. Ogura, S. Orimo, A. Sagisaka et al., Phys. Rev. E 77, 016401 (2008).

[17] I. Hofmann, J. Meyer-ter-Vehn, X. Q. Yan, A. Orzhekhovskaya, and S. Yaramyshev, Phys. Rev. Accel. Beams 14, 031304 (2011).

[18] S. D. Kraft, C. Richter, K. Zeil, M. Baumann, E. Beyreuther, S. Bock, M. Bussmann, T. E. Cowan, Y. Dammene, W. Enghardt, U. Helbig, L. Karsch et al., New J. Phys. 12, 085003 (2010).

[19] D. Jung, L. Yin, B. J. Albright, D. C. Gautier, R. Hörlein, D. Kiefer, A. Henig, R. Johnson, S. Letzring, S. Palaniyappan, R. Shah, T. Shimada, X. Q. Yan et al., Phys. Rev. Lett. 107, 115002 (2011).

[20] U. Linz and J. Alonso, Phys. Rev. Accel. Beams 19, 124802 (2016).

[21] L. Brouwer, S. Caspi, K. Edwards, A. Godeke, and S. Prestemon, Nucl. Instrum. Methods Phys. Res., Sect. A 957, 163414 (2020).

[22] D. Uriot and N. Pichoff, in Proceedings of the 6th International Particle Accelerator Conference (IPAC'15), Richmond, VA, USA (JACoW, Geneva, 2015), pp. 92-94. 
[23] A. A. Morris, arXiv:2006.13117v1.

[24] M. J. Wu, J. G. Zhu, D. Y. Li, T. Yang, Q. Liao, Y. X. Geng, X. H. Xu, C. C. Li, Y. R. Shou, Y. Y. Zhao, Y. R. Lu, H. Y. Lu, W. J. Ma, C. Lin, K. Zhu, and X. Q. Yan, Nucl. Instrum. Methods Phys. Res., Sect. A 955, 163249 (2020).

[25] R. H. Siemann, Phys. Rev. Accel. Beams 11, 050003 (2008).

[26] I. Hofmann, J. Meyer-ter-Vehn, X. Q. Yan, and H. Al-Omari, Nucl. Instrum. Methods Phys. Res., Sect. A 681, 44 (2012).

[27] S. Y. Lee, Accelerator Physics, 2nd ed. (World Scientific, Singapore, 2004).

[28] N. Tsoupas, D. Kayran, V. Litvinenko, and W. W. Mackay, in Proceedings of the 2011 Particle Accelerator Conference (PAC'11) (IEEE, New York, NY, USA, 2011), pp. 2163-2165.

[29] N. Tsoupas, D. Kayran, and V. Litvinenko, Phys. Procedia 66, 376 (2015).
[30] R. P. Walker, M. Apollonio, C. P. Bailey, R. Bartolini, M. P. Cox, R. T. Fielder, N. P. Hammond, M. T. Heron, J. Kay, I. P. S. Martin et al., in Proceedings of the 5th International Particle Accelerator Conference (IPAC'14), Dresden, Germany (JACoW, Geneva, 2014), pp. 331-333.

[31] L. N. Brouwer, Canted-cosine-theta superconducting accelerator magnets for high energy physics and ion beam cancer therapy, Ph.D. thesis, UC Berkeley, 2015.

[32] W. S. Wan, L. Brouwer, S. Caspi, S. Prestemon, A. Gerbershagen, J. M. Schippers, and D. Robin, Phys. Rev. Accel. Beams 18, 103501 (2015).

Correction: The byline footnote indicator intended for the second author was placed incorrectly during production and has been fixed. 